\title{
COMMUNICATION
}

\section{Le chirurgien oral au cœur du diagnostic de sarcome d'Ewing : A propos d'un cas}

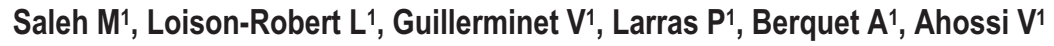

1. CHU Dijon

\section{Introduction}

Le sarcome d'Ewing est une tumeur maligne primitive osseuse (ou extra osseuse dans $20 \%$ des cas) qui atteint les enfants et les jeunes adolescents dans les deux premières décennies de la vie. II a été décrit par James Ewing pour la première fois en 1921. II ne représente que $2 \%$ des tumeurs pédiatriques mais c'est la deuxième tumeur osseuse la plus fréquente dans cette population. II atteint principalement les os plats et dans seulement moins de $2 \%$ des cas les os de la face, comme la mandibule. Le diagnostic est peu aisé cliniquement du fait de l'absence de signe spécifique. Le plus souvent il n'existe qu'une masse palpable voire aucun symptôme. II repose uniquement sur un examen cytogénétique mettant en évidence une translocation spécifique impliquant le gène EWSR1 sur une biopsie tumorale. Le traitement repose sur une séquence incluant chimiothérapie, chirurgie carcinologique puis radiothérapie.

\section{Observation}

Le cas présenté est celui d'une petite fille de 4 ans adressée au service d'Odontologie du CHU de Dijon initialement pour prise en charge d'une cellulite d'origine dentaire sans altération de l'etat général. A l'examen extra oral, elle présentait une tuméfaction génienne basse légèrement douloureuse. L'examen endo buccal mettait en evidence une mobilité récente des dents du secteur incisivo canin mandibulaire et une muqueuse gingivale bourgeonnante au niveau de la dent numéro 71 tombée quelques jours auparavant. Un aspect de lyse osseuse intéressant la symphyse mandibulaire était observé sur l'orthopantomogramme. Cet aspect atypique à l'imagerie a conduit à une biopsie osseuse en urgence sous anesthésie générale ; ce qui a permis de conclure rapidement au diagnostic de sarcome d'Ewing mandibulaire. Une prise en charge médicale adaptée en milieu spécialisé a pu ainsi être débutée sans délai.

\section{Discussion}

La littérature rapporte d'autres cas similaires qui ont également été diagnostiqués à tort comme une pathologie infectieuse d'origine dentaire.

\section{Conclusion}

Le but de ce cas est de montrer que le diagnostic de sarcome d'Ewing doit être évoqué devant toute lésion atypique osseuse mandibulaire de l'enfant. Les médecins et chirurgiens dentistes spécialisés en chirurgie orale sont alors au premier plan pour permettre d'établir un diagnostic précoce et assurer une prise en charge rapide en limitant ainsi les séquelles fonctionnelles et esthétiques liées à la localisation.

salehmyriam99@hotmail.fr

(C) The authors, published by EDP Sciences. This is an Open Access article distributed under the terms of the Creative Commons Attribution License 4.0 (http://creativecommons.org/licenses/by/4.0/). 